\title{
Similarity law for the streamwise turbulence intensity in zero-pressure- gradient turbulent boundary layers
}

\author{
I. Marusic, A. K. M. Uddin, ${ }^{\text {a) }}$ and A. E. Perry \\ Department of Mechanical and Manufacturing Engineering, University of Melbourne, Parkville, Victoria \\ 3052, Australia
}

(Received 3 January 1997; accepted 6 August 1997)

\begin{abstract}
A similarity relationship is proposed to describe the streamwise broadband-turbulence intensity in a zero-pressure-gradient boundary layer. The formulation is applicable to the entire region of the flow beyond the viscous buffer zone and is based on the attached eddy hypothesis, the Reynolds-number-similarity hypothesis and the assumed existence of Kolmogorov eddies with a universal inertial subrange. Experimental data of the authors and those from various published works covering a large Reynolds number range are investigated in light of this formulation. (C) 1997 American Institute of Physics. [S1070-6631(97)00212-2]
\end{abstract}

\section{INTRODUCTION}

Even though the zero-pressure-gradient turbulent boundary layer is often referred to as the canonical boundary layer, comprehensive turbulence measurements still remain sparse, particularly over an extensive range of Reynolds numbers. However, over the past decade a considerable range of measurements have become available for, what is relatively the easiest component to measure of the kinematic Reynolds stresses, the streamwise broadband-turbulence intensity $\left(\overline{u_{1}^{2}}\right)$. In recent reviews of some of these experiments by Gad-el-Hak and Bandyopadhyay ${ }^{1}$ and Fernholz and Finley ${ }^{2}$ the $\overline{u_{1}^{2}}$ data has been described with special mention of strong Reynolds number effects when the data is presented normalised using inner-wall variables. Unfortunately, as yet no theoretical framework has been agreed on with which to describe these so-called Reynolds number effects and questions such as the applicability of inner and outer scaling regions still appear to be points of contention. By inner scaling we mean $\overline{u_{1}^{2}} / U_{\tau}^{2}=f\left[z_{+}\right]$while outer scaling involves normalisation with length scale $\delta_{c}$, taken here to be the boundary layer thickness, i.e., $\overline{u_{1}^{2}} / U_{\tau}^{2}=\phi\left[z / \delta_{c}\right]$. Here overbars denote temporal averages and $u_{1}$ is the streamwise component of the fluctuating velocity, $U_{\tau}$ is the wall shear velocity, $z_{+}=z U_{\tau} / \nu$, where $z$ is the distance normal to the wall and $\nu$ is the kinematic viscosity of the fluid.

With regard to the applicability of inner-flow scaling, Mochizuki and Nieuwstadt ${ }^{3}$ surveyed a large collection of experimental data in both conventional flat plate boundary layers and in pipes and ducts. Specific attention was given to whether the peak in $\overline{u_{1}^{2}}$ which occurs at approximately $z_{+}=15$ is Reynolds number independent. Variations between different experiments of this value for $\overline{u_{1}^{2}} / U_{\tau}^{2}$ were $\pm 10 \%$ but the variation was concluded not to be statistically significant. Coles ${ }^{4}$ also surveyed a large numbers of wallbounded flow experiments and arrived tentatively at the same conclusion. Fernholz and Finley ${ }^{2}$ suggest that a weak Reynolds number effect may be present but like Coles, draw attention to the difficulty of reaching firm conclusions in the

\footnotetext{
${ }^{a)}$ Present address: Department of Mechanical Engineering, Bangladesh University of Engineering and Technology, Dhaka 1000, Bangladesh.
}

presence of the significant scatter in the data. Mochizuki and Nieuwstadt further conclude that $\overline{u_{1}^{2}}$ does scale with innerflow variables in the entire wall region (we understand this to be from $z_{+}=0$ to $z / \delta_{c} \leqslant 0.15$, say) which is in support of the approach taken by many conventional computational turbulence models which assume the existence of inner-layer scaling for the Reynolds stresses and mean-flow velocity. Smits and Dussauge ${ }^{5}$ and Dussauge et al. ${ }^{6}$ also reviewed the data presented by Fernholz and Finley ${ }^{2}$ and arrived at the conclusion that for a high enough Reynolds number the $\overline{u_{1}^{2}}$ profiles display similarity in the viscous sublayer and buffer layer in inner scaling, while similarity in outer scaling is observed in the mean-flow logarithmic layer and the remainder of the outer region.

Doubts as to the validity of inner-flow scaling in the near-wall region have recently been raised by Bradshaw and Huang ${ }^{7}$ from studies of computational DNS results. Durst, Jovanovic and Sender ${ }^{8}$ made careful LDA measurements in a low-Reynolds-number pipe flow and also conclude that turbulence intensities in the wall region do not scale with inner variables. However, they do state that very close to the wall only a weak Reynolds number dependence exists. Below $z_{+}=15$ they believe their data, within experimental uncertainty, does scale with inner variables.

In this paper we will consider turbulence measurements covering a large range of Reynolds number including new data by the authors and experiments not reviewed previously. This new data fills in many gaps in the Reynolds number range covered by Fernholz and Finley ${ }^{2}$ and Smits and Dussauge. ${ }^{5}$ We are primarily concerned with proposing a formulation to describe the flow beyond the viscous buffer zone, that is the outer-scaling region mentioned by Smits and Dussauge. Previous studies by Perry, Lim and Henbest ${ }^{9}$ and Perry and $\mathrm{Li}^{10}$ have argued in favor of this type of outerscaling region and formulations were proposed to describe how the broadband turbulence intensities and Reynolds shear stress behave but this was restricted to the region of the layer where only the mean logarithmic profile applies, that is, in the turbulent wall region, defined tentatively here as $z_{+} \geqslant 100$ and $z / \delta_{c} \leqslant 0.15$. In this paper we will extend this formulation to the entire outer region of the flow, i.e., $z_{+} \geqslant 100$ and 
$z / \delta_{c} \leqslant 1$, and attempt to include Reynolds number effects in the Perry and Li formulation which is based effectively on the inviscid attached eddy model, together with Kolmogorov eddies and an anisotropic viscous cut-off. The final part of the paper will consider the implications of the proposed formulation on flow in the region $z_{+}<100$. Particular attention will be given to Townsend' $\mathrm{s}^{11,12}$ attached eddy hypothesis and the consequent attached eddy model, most recently used by Perry and Marusic ${ }^{13}$ and Marusic and Perry ${ }^{14}$ to describe flows with arbitrary pressure gradients.

\section{PREVIOUS FORMULATIONS FOR THE TURBULENT WALL REGION}

Townsend ${ }^{12}$ using the attached eddy hypothesis showed that $\overline{u_{1}^{2}}$ will have a logarithmic relationship in the region of the flow where $\delta_{1} \ll z \ll \delta_{c}$, where $\delta_{1}$ and $\delta_{c}$ are the length scales of the smallest and largest attached eddies in the flow, respectively. This effectively relies on a large Reynolds number existing since if we take $\delta_{1}=0\left(100 \nu / U_{\tau}\right)$ (the Kline et al. ${ }^{15}$ scaling) then the ratio of the largest to smallest attached eddy is proportional to the Karman number $K_{\tau}=\delta_{c} U_{\tau} / \nu$. Townsend's hypothesis was formalised into a crude physical model for wall-turbulence initially by Perry and Chong. ${ }^{16}$ The salient feature of this model is that the boundary layer beyond the viscous buffer zone is described by an assemblage of statistically representative eddies of varying length scales with varying population density of eddies per unit eddy length scale. For full details of the model the reader is referred to sections 3 and 4 of Perry and Marusic $^{13}$ where the above mentioned logarithmic behavior is also fully derived.

Perry, Henbest and Chong ${ }^{17}$ following the dimensional scaling arguments of Perry and Abell ${ }^{18}$ for spectra, which are consistent with the attached eddy hypothesis, deduced that for negligible contributions from small-scale viscosity dependent motions in the turbulent wall region,

$$
\frac{\overline{u_{1}^{2}}}{U_{\tau}^{2}}=B_{1}-A_{1} \ln \left[\frac{z}{\delta_{c}}\right]-C\left(z_{+}\right)^{-1 / 2},
$$

where $A_{1}$ and $C$ are universal constants and $B_{1}$ is a largescale characteristic constant. The last term in (1) represents a viscous correction due to the viscous cut-off of the Kolmogorov motions and is valid for conditions of local isotropy, sufficiently high Reynolds number and sufficiently high $z_{+}$ and is also based on the assumption of Townsend ${ }^{11}$ that local production and dissipation of energy are in balance in the turbulent wall region. Perry and $\mathrm{Li}^{10}$ incorporated a more general viscous correction term in (1) and obtained

$$
\frac{\overline{u_{1}^{2}}}{U_{\tau}^{2}}=B_{1}-A_{1} \ln \left[\frac{z}{\delta_{c}}\right]-V\left[z_{+}\right],
$$

where $V\left[z_{+}\right]$is the viscous correction term and assumes local isotropy and can be applied down to moderate Reynolds numbers. By applying the Kovasznay ${ }^{19}$ spectrum formula beyond $k_{1} z=2.5$, Perry et al. ${ }^{20}$ arrived at the following form:

$$
\begin{aligned}
V\left[z_{+}\right]= & 5.58\left(z_{+}\right)^{-1 / 2}-22.4\left(z_{+}\right)^{-1}+22.0\left(z_{+}\right)^{-5 / 4} \\
& -5.62\left(z_{+}\right)^{-2}+1.27\left(z_{+}\right)^{-11 / 4} .
\end{aligned}
$$

Spalart ${ }^{21}$ proposed a simpler expression of the form

$$
V\left[z_{+}\right]=4.37\left(z_{+}\right)^{-1 / 2} .
$$

Both (3) and (4) have the correct asymptotic behavior in the turbulent wall region as the Reynolds number goes to infinity and $z_{+}$is sufficiently large.

Using $V\left[z_{+}\right]$as formulated by (3) with $A_{1}=1.03$ and $B_{1}=2.39$, Perry and $\mathrm{Li}^{10}$ found that their results were in a good agreement with (2) except for $z_{+}$less than about 200 where the experimental data showed more spread than the predictive equation. Perry and $\mathrm{Li}^{10}$ attributed this to the approximation involved in the Kovasznay ${ }^{19}$ spectrum.

Perry and Li proposed an alternative numerical method for determining $V\left[z_{+}\right]$which, recently, Hafez (private communication) described in an algebraic form as

$$
V\left[z_{+}\right]=5.58\left\{1-z_{+}^{-0.9}\right\} z_{+}^{-1 / 2} .
$$

In the subsequent analysis this functional form of $V\left[z_{+}\right]$, which is probably valid for $z_{+} \geqslant 200$, will be used.

$\mathrm{Hafez}^{22}$ found that for low-Reynolds-number zeropressure-gradient flows and for layers in favourablepressure-gradient, (2) needs to be modified. It is further necessary to incorporate a liberally defined turbulent wall region (i.e., $z_{+} \geqslant 50$ and $z / \delta_{c} \leqslant 0.30$ ) as opposed to the conservative definitions of Perry et al. ${ }^{17,9}\left(z_{+} \geqslant 100 ; z / \delta_{c} \leqslant 0.15\right)$. From the work of Hafez, ${ }^{22}$ Perry et al. ${ }^{23}$ and Perry and Marusic, ${ }^{13}$ it is known that the characteristic constant $B_{1}$ in (2) should be a function of Coles $^{24}$ wake factor $\Pi$ for equilibrium and quasi-equilibrium layers. On the basis of a limited range of experimental data for zero and favourable pressure gradients at low Reynolds numbers, $\mathrm{Hafez}^{22}$ expressed (2) as

$$
\frac{\overline{u_{1}^{2}}}{U_{\tau}^{2}}=B_{1}[\Pi]-A_{1} \ln \left[\frac{z}{\delta_{c}}\right]-V\left[z_{+}\right],
$$

where tentatively

$$
B_{1}[\Pi]=0.41+3.66 \Pi-0.76 \Pi^{2} .
$$

\section{BROADBAND TURBULENCE INTENSITY RESULTS}

The experiments were performed in two nominally zero streamwise pressure gradient flows with freestream velocities $U_{\infty}$ set at nominally $10 \mathrm{~m} / \mathrm{s}$ and $30 \mathrm{~m} / \mathrm{s}$ giving $R_{\theta}$ values of 4140 and 11928 , respectively $\left(R_{\theta}=\theta U_{1} / \nu\right.$, where $\theta$ is the momentum thickness and $U_{1}$ is the freestream velocity). Dynamically calibrated single wire hot-wires were used for the turbulence measurements as described by Perry. ${ }^{25}$ Pitot-static tubes and hot-wires were used to measure mean-flow profiles and showed excellent agreement. All measurements techniques and procedures are similar to those used in Marusic and Perry ${ }^{14}$ and full details are given in Uddin. ${ }^{26}$

The mean-flow integral parameters for the two flow cases are tabulated in Table I. The wall-shear velocity $U_{\tau}$ was evaluated using the Clauser chart method with the log- 
TABLE I. Mean flow boundary layer parameters.

\begin{tabular}{cccccccccc}
\hline \hline $\begin{array}{c}\text { Flow } \\
\text { case }\end{array}$ & $\begin{array}{c}U_{\infty} \\
\mathrm{m} / \mathrm{s}\end{array}$ & $\begin{array}{c}U_{1} \\
\mathrm{~m} / \mathrm{s}\end{array}$ & $\begin{array}{c}\theta \\
\mathrm{mm}\end{array}$ & $\begin{array}{c}\delta^{*} \\
\mathrm{~mm}\end{array}$ & $\begin{array}{c}\delta_{c} \\
\mathrm{~mm}\end{array}$ & $\begin{array}{c}U_{\tau} \\
\mathrm{m} / \mathrm{s}\end{array}$ & $\Pi$ & $R_{\theta}$ & $K_{\tau}$ \\
\hline 1 & 10 & 9.88 & 6.28 & 8.73 & 64.28 & 0.38 & 0.57 & 4140 & 1655 \\
2 & 30 & 30.31 & 6.11 & 8.25 & 67.62 & 1.07 & 0.55 & 11928 & 4704 \\
\hline \hline
\end{tabular}

law constants 0.41 and 5.0. The boundary layer thickness $\delta_{c}$ was determined by an integral method as outlined by Perry and Li. ${ }^{10}$ Here

$$
\delta_{c}=\frac{\delta^{*}}{C_{1}} \frac{U_{1}}{U_{\tau}},
$$

where $\delta^{*}$ is the displacement thickness and

$$
C_{1}=\int_{0}^{1} U_{D}^{*}[\eta] d \eta
$$

where $U_{D}^{*}=\left(U_{1}-U\right) / U_{\tau}$ and $\eta=z / \delta_{c} . C_{1}$ is evaluated using an expression for Coles' ${ }^{24}$ law of the wall and law of the wake as given by Perry and Marusic ${ }^{13}$ for $U_{D}^{*}[\eta]$.

The experimental streamwise broadband-turbulence intensity profiles are shown with outer-flow scaling and innerflow scaling in Figs. 1(a) and 1(b), respectively. The predicted $\overline{u_{1}^{2}} / U_{\tau}^{2}$ values using (6) along with (7) and (5) for calculating $B_{1}[\Pi]$ and $V\left[z_{+}\right]$, are also shown in the figure. Expression (6) shows agreement with the data nominally in the inclusive range $z_{+}>180$ and $z / \delta_{c}<0.20$ for $R_{\theta}=4140$ and $z_{+}>350$ and $z / \delta_{c}<0.20$ for $R_{\theta}=11928$. If we take the turbulent wall region to be in the range $z_{+} \geqslant 100$ and $z / \delta_{c} \leqslant 0.15$ as determined from mean-flow data, then (6) agrees reasonably well with the outer limit but for $z_{+} \approx 100$ the viscous cut-off function $V\left[z_{+}\right]$seems to be inadequate in explaining the deviation from the log-law. This deviation could well be due to an "attached eddy cut-off" and this issue will be discussed further in the following section. Alternatively, or in addition to this, the deviation could also be attributed to a much larger $V\left[z_{+}\right]$cut-off than predicted by (3) or (5) due to the dissipation range becoming highly anisotropic.

In the outer region of the flow, the two sets of data in Fig. 1(a) show good agreement suggesting that $z / \delta_{c}$ is the only important parameter. This behavior is consistent with Townsend's $^{27}$ Reynolds-number-similarity hypothesis, that is: all mean-relative motions and energy-containing components of the turbulence are independent of the fluid viscosity when expressed in terms of the variables in Fig. 1(a). Also it needs to be assumed that zero-pressure-gradient layers are self-preserving to a good approximation. Other studies have also supported this conjecture, including Fernholz and Finley $^{2}$ and Antonia et al. ${ }^{28}$ who suggested that the Reynolds-number dependence of the large-scale structures diminishes beyond $R_{\theta} \approx 3000$. Perry et al. ${ }^{17}$ showed strong support for this outer-flow behavior in fully-developed turbulent pipe flow.

The extent of the outer-flow scaling collapse will depend on the Reynolds number of the flow, i.e. the higher the Reynolds number the lower the value of $z / \delta_{c}$ at which the deviation from the collapse occurs. Figure 2 shows a collection
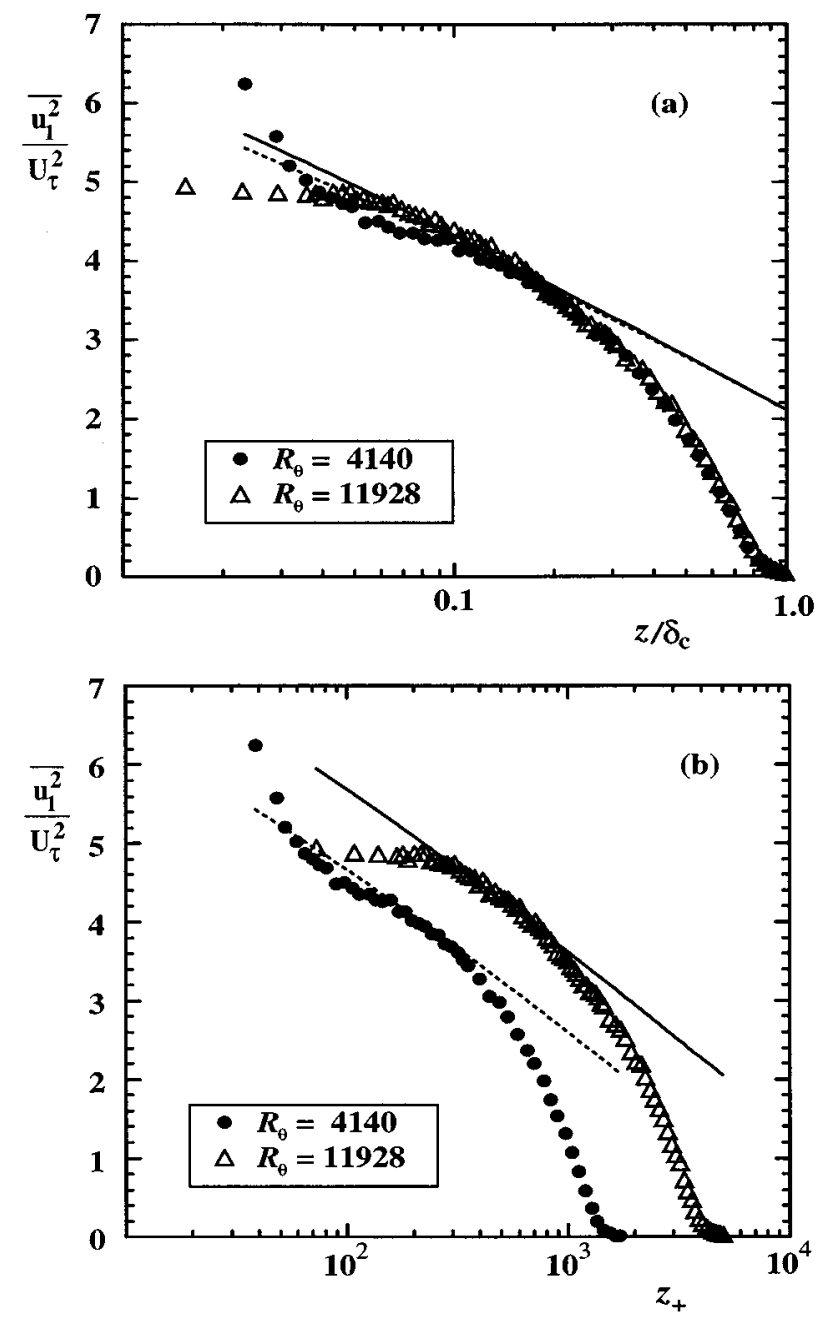

FIG. 1. Streamwise broadband turbulence intensity profiles at $R_{\theta}=4140$ and $11928\left(K_{\tau}=1655\right.$ and 4704). The dotted line and the solid line represent (6) for $R_{\theta}=4140$ and 11928 respectively. (a) Outer-flow scaling, (b) inner-flow scaling. Note that $A_{1}=1.03$; and $B_{1}$ and $V\left[z_{+}\right]$are calculated using (7) and (5), respectively.

of data covering a $R_{\theta}$ range of 1,000-13,000. The data shown in Fig. 2 are all for similar experimental conditions all using the same hot-wire techniques and dynamic calibration as described by Perry. ${ }^{25}$ Note that in this figure the solid line and the dotted line represent (6) with $V\left[z_{+}\right]=0$ for $R_{\theta}$ $=11928(\Pi=0.55)$ and $R_{\theta}=1003(\Pi=0.29)$ respectively. In these cases, $A_{1}=1.03$ and $B_{1}$ is calculated using (7). The figure shows an outer-flow scaling collapse of the data for $R_{\theta}>2788$ and a systematic peel-off from the "universal" curve occurs as the wall is approached. The extent of the collapse to the universal curve increases with increasing Reynolds number. Considering the value of $z / \delta_{c}$ at which this peel-off is occurring in wall units, i.e., $z_{+}$, it appears that the departure from the universal curve occurs at $z+$ less than approximately 500. This may explain why the data of Erm and Joubert ${ }^{29}$ corresponding to $K_{\tau}=547\left(R_{\theta}=1003\right)$ does not agree with the universal curve. Overall, the figure does seem to indicate that as the Reynolds number increases, the streamwise broadband-turbulence-intensity profiles collapse with outer-flow scaling with the extent of collapse in- 


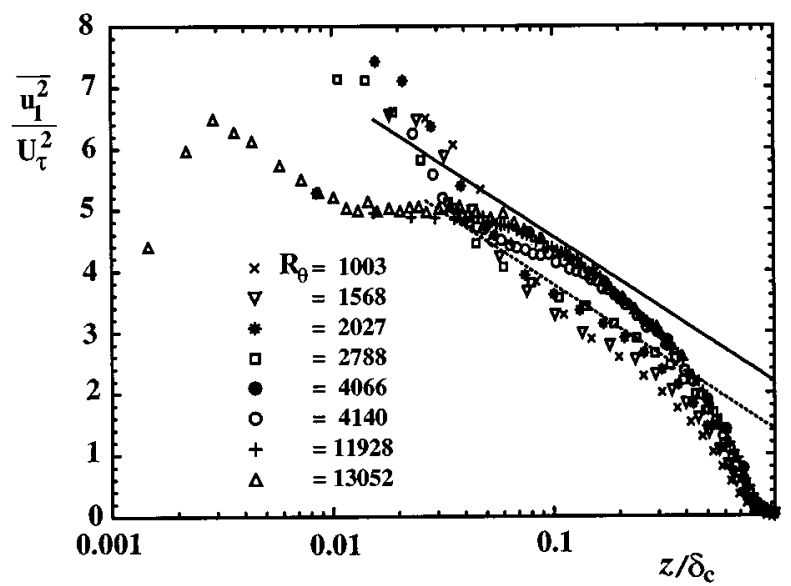

FIG. 2. Comparison of the streamwise broadband turbulence intensity profiles for various $R_{\theta}$. The symbols represent the experimental data from various sources (Please see the Appendix for the sources). The solid line and the dotted line represent (6) with $V\left[z_{+}\right]=0$ for $R_{\theta}=11928(\Pi=0.55)$ and $R_{\theta}=1003(\Pi=0.29)$ respectively. Note that $A_{1}=1.03$ and $B_{1}$ is calculated using (7) to take into account the variation of $B_{1}$ with $\Pi$.

creasing and propagating towards the wall with increasing Reynolds number. Additional data from various sources to be presented in the following section, add weight to this conclusion.

\section{EXTENDED FORMULATION}

In this section, an extended framework is presented which summarises previous findings and includes the entire region of the flow above the mean-flow buffer zone, i.e., $z_{+} \geqslant 100$. This is done by first considering an analogy with the mean-flow velocity-defect and the streamwise broadband-turbulence intensity at sufficiently large Reynolds numbers.

On a semi-logarithmic plot, both the mean-flow velocity defect and $\overline{u_{1}^{2}} / U_{\tau}^{2}$ show a straight line region in the middle part of the plot with deviations at both ends. In the case of the mean-flow velocity defect, examples of which can be found in the review of Fernholz and Finley, ${ }^{2}$ the deviation near the wall is attributed to viscous effects while the outer part is referred to as the wake region. An analogous picture can be imagined for the streamwise broadband-turbulence intensity. This is represented schematically in Fig. 3. As with the mean flow, the deviation from the logarithmic profile near the wall is attributed to viscous effects and will be referred to as a viscous deviation $V_{g}$. The deviation in the outer part of the boundary layer is due to wake effects and this deficit will be referred to as a wake deviation $W_{g}$ which, from Townsend's Reynolds-number-similarity hypothesis, is a function of $z / \delta_{c}$ alone for constant $\Pi$.

With this analogy, (6) can be rewritten as

$$
\frac{\overline{u_{1}^{2}}}{U_{\tau}^{2}}=B_{1}-A_{1} \ln \left[\frac{z}{\delta_{c}}\right]-V_{g}\left[z_{+}, \frac{z}{\delta_{c}}\right]-W_{g}\left[\frac{z}{\delta_{c}}\right] \text {. }
$$

Here, the viscous deviation $V_{g}$ is proposed to be made up of two parts. One part is the viscous correction $V\left[z_{+}\right]$which is assumed to be a Kolmogorov eddy-type cut-off and is thus isotropic in nature and the second part contributes to the

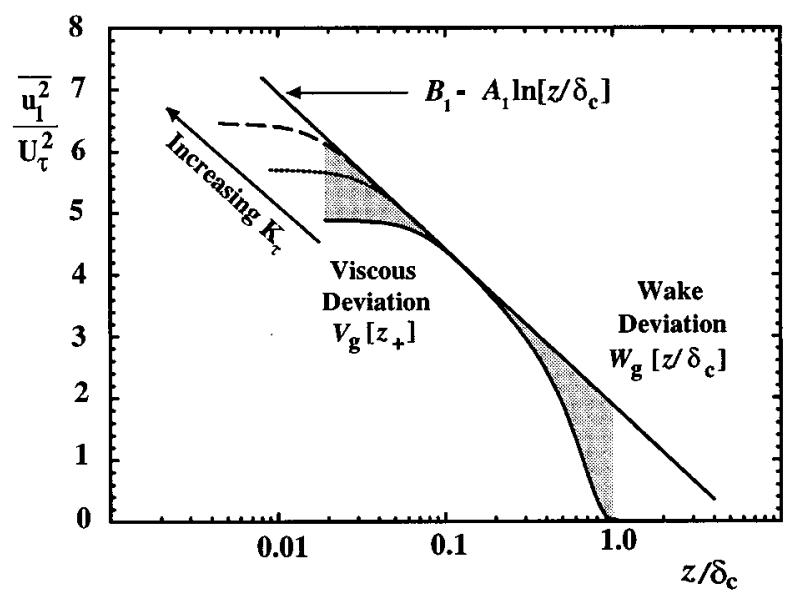

FIG. 3. A mean-flow velocity-defect analogous picture for the streamwise broadband-turbulence intensity for flow above the viscous buffer zone $\left(z_{+} \geqslant 100\right)$.

anisotropic behavior as we approach the boundary. The anisotropic correction is believed to be made up of the cut-off of anisotropic dissipating motions and an attached eddy cutoff. The effect of the attached eddy cut-off is to give a levelling off of $\overline{u_{1}^{2}} / U_{\tau}^{2}$ and the beginning of the drop off in $-\overline{u_{1} u_{3}} / U_{\tau}^{2}$ (see Perry and Marusic ${ }^{13}$ ). All of this occurs near the outer edge of the buffer zone $\left(z_{+} \approx 60\right)$ and is simply the consequence of there being no representative attached eddy scales below the smallest scale $\delta_{1}=O\left(100 \nu / U_{\tau}\right)$.

Therefore, considering the trends of $\overline{u_{1}^{2}}$ profiles at larger Reynolds numbers, a function of the form

$$
V_{g}=\underbrace{S_{A}\left[z_{+}\right]\left(E_{1}-A_{1} \ln \left[z_{+}\right]\right)}_{\text {anisotropic cut-off }}+\underbrace{S_{V}\left[z_{+}\right] V\left[z_{+}\right]\left(1-z / \delta_{c}\right)}_{\text {isotropic Kolmogorov cut-off }}
$$

is proposed, where $E_{1}$ is a constant and $S_{A}\left[z_{+}\right]$and $S_{V}\left[z_{+}\right]$ are two switching functions. Note that the functional form of $V\left[z_{+}\right]$is given by (5). The first function on the right hand side of (9) accounts for the anisotropic cut-off; whereas the second function takes care of the Kolmogorov cut-off and this will have a form and asymptotic behavior at large $z_{+}$ similar to the viscous correction function given by (5). As a first approximation, a linear factor $\left(1-z / \delta_{c}\right)$ multiplies the $V\left[z_{+}\right]$term. Strictly, the functional form of $V\left[z_{+}\right]$given earlier is valid only in the turbulent wall region and we have no firm idea of what its functional form is in the outer part of the layer since the assumption of local production balancing local dissipation breaks down. From a study of the spectral data beyond the turbulent wall region measured by Perry and $\mathrm{Li}^{10}{ }^{10}$ it appears that $V\left[z_{+}\right]$should be less than that predicted by the turbulent wall region value for the same $z_{+}$. We have decided to incorporate the linear factor for convenience but suspect that the truth lies somewhere in between $V\left[z_{+}\right]$and $V\left[z_{+}\right]\left(1-z / \delta_{c}\right)$.

The switching functions $S_{A}\left[z_{+}\right]$and $S_{V}\left[z_{+}\right]$ensure that

$$
V_{g} \rightarrow\left\{\begin{array}{ll}
C z_{+}^{-1 / 2} & \text { as } z_{+} \rightarrow \infty \\
E_{1}-A_{1} \ln \left[z_{+}\right] & \text {as } z_{+} \rightarrow O(100)
\end{array},\right.
$$


TABLE II. Values of various constants in equations (8), (9), (11) and (12).

\begin{tabular}{ccc}
\hline \hline Constant & Numerical value & Referencing equation \\
\hline$A_{1}$ & 1.03 & 9 and 8 \\
$B_{1}$ & 2.39 & 8 \\
$m_{A}$ & 0.0021 & 11 \\
$m_{V}$ & 0.0059 & 12 \\
$E_{1}$ & 6.25 & 9 \\
\hline
\end{tabular}

where $C$ is a universal constant. Given these constraints, a possible form of the switching functions may be given by

$$
\begin{aligned}
& S_{A}\left[z_{+}\right]=\operatorname{erfc}\left[m_{A} z_{+}\right], \\
& S_{V}\left[z_{+}\right]=\operatorname{erf}\left[m_{V} z_{+}\right],
\end{aligned}
$$

where $m_{A}$ and $m_{V}$ are constants; the numerical values of these constants can be found from a curve-fitting procedure for one data set and then must apply to all the other flows since $V_{g}$ is assumed to be universal. The form of the switching functions $S_{A}$ and $S_{V}$ chosen here are quite arbitrary but do seem to fit the data reasonably well.

In the case of $W_{g}$, this is taken to be a universal function of $z / \delta_{c}$ as required by Townsend's Reynolds-numbersimilarity hypothesis and the usual approximate selfpreserving flow assumption for zero-pressure-gradient layers. $W_{g}$ could be computed numerically using a model developed by Perry and Marusic ${ }^{13}$ and Marusic and Perry ${ }^{14}$ but this would be very complicated and would depend on the assumed shape of the representative eddies. For simplicity, an analytical functional form is chosen which involves the same nondimensional variables as the attached eddy model would give. This is

$$
W_{g}[\eta]=B_{1} \eta^{2}(3-2 \eta)-A_{1} \eta^{2}(1-\eta)(1-2 \eta) .
$$

The polynomial terms are identical to the expressions used by Lewkowicz ${ }^{30}$ to describe the mean-flow wake function and the coefficients are determined from the boundary conditions at the edge of the layer and in the wall region, i.e., $\overline{u_{1}^{2}} / U_{\tau}^{2}=0 ; \partial\left(\overline{u_{1}^{2}} / U_{\tau}^{2}\right) / \partial\left(z / \delta_{c}\right)=0$ at $z / \delta_{c}=1$ and $W_{g}=0$ as $z / \delta_{c} \rightarrow 0$. A summary of the constants used in (8), (9), (11) and (12) are given in Table II. The values for $A_{1}$ and $B_{1}$ shown are as given by Perry and Li. ${ }^{10}$

It should be noted that the details of these curve fits are of secondary importance, which most likely will be subject to alteration and further refinement. What is important is the similarity scaling behavior which this formulation expresses, namely, a viscous region with lengths scaling with $\nu / U_{\tau}$ which smoothly blends into a region where the lengths scale with $\delta_{c}$ alone if the Reynolds number is sufficiently high.

Figure 4 shows the curve fit to the data corresponding to $R_{\theta}=11928(\Pi \approx 0.55)$ together with the individual components which make up equation (8). The figure illustrates the purpose of the switching functions in moving from one asymptotic relation to another. Using the constants thus obtained and summarised in Table II, equation (8) is compared to other experimental data in Fig. 5. The data sets in Fig. 5 have been chosen to present a large spread in Reynolds number and include DNS numerical data together with hot-wire (HWA) and LDA measurements. See the Appendix. The

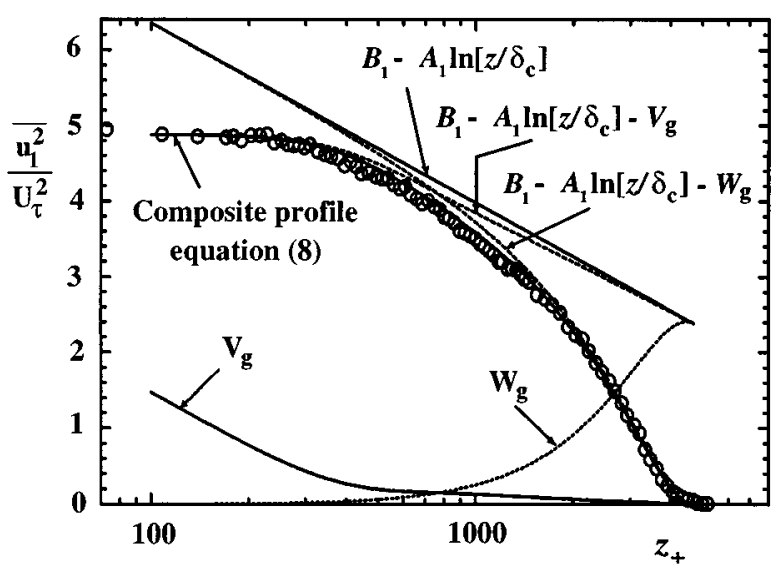

FIG. 4. Individual components which make up equation (8) compared to experimental data at $R_{\theta}=11928$.

similarity equation (8) is seen to fit the data reasonably well for the moderate and high Reynolds number flows. If the two length scales $\nu / U_{\tau}$ and $\delta_{c}$ are sufficiently separated in value, then the formulation is applicable for $z_{+} \geqslant 100$ to $z / \delta_{c} \leqslant 1$. For low Reynolds numbers, i.e., $K_{\tau}<2500$ say, i.e., $R_{\theta}<6000$ approximately, the two length scales are not sufficiently separated and the lower limit of applicability of $z_{+}$ increases as the Reynolds number decreases, as can be clearly seen in Fig. 5(a). We have no formulation for this low-Reynolds-number effect but for the data shown, the data still fits in the wake region rather well.

It is worth noting that Fig. 5(a) looks very similar to figure 5 of Phillips ${ }^{31}$ who deduced the functional form for the Reynolds stresses from matched asymptotic expansion methods. The authors agree broadly with the results of Phillips but not necessarily with details such as numerical values of constants or the behavior of the normal to the wall component of turbulence intensity (which is not treated here but is discussed in Perry et al. ${ }^{17}$ and Perry and Marusic ${ }^{13}$ ).

The results in Fig. 5(a) are re-plotted versus $z / \delta_{c}$ in Fig. 5 (b). Here, the "peel-off' from the outer-flow scaling collapse is clearly seen with decreasing Reynolds number.

\section{DISCUSSION}

\section{A. Near-wall region}

The peak in intensity at $z_{+} \approx 15$ can be clearly seen in Fig. 5(a) and we conjecture that this may have something to do with the early formation process of the attached eddies. Very little is understood about this region. As stated earlier, the similarity relation (8) does not extend into the viscous buffer zone $\left(z_{+}<100\right)$ where as seen in Fig. 5(a) there would appear to be a trend towards inner-flow scaling. As discussed in Sec. I, the question of whether or not inner-flow scaling applies in this region is still a point of contention and most likely will remain so until reliable well-resolved nearwall measurements can be made. The pipe-flow LDA measurements of Durst et al. ${ }^{8}$ using a glass wall with a matched reflective-index fluid are a positive development and an error analysis shows these results to be accurate and agreement with DNS data gives further confidence in the measurements. 


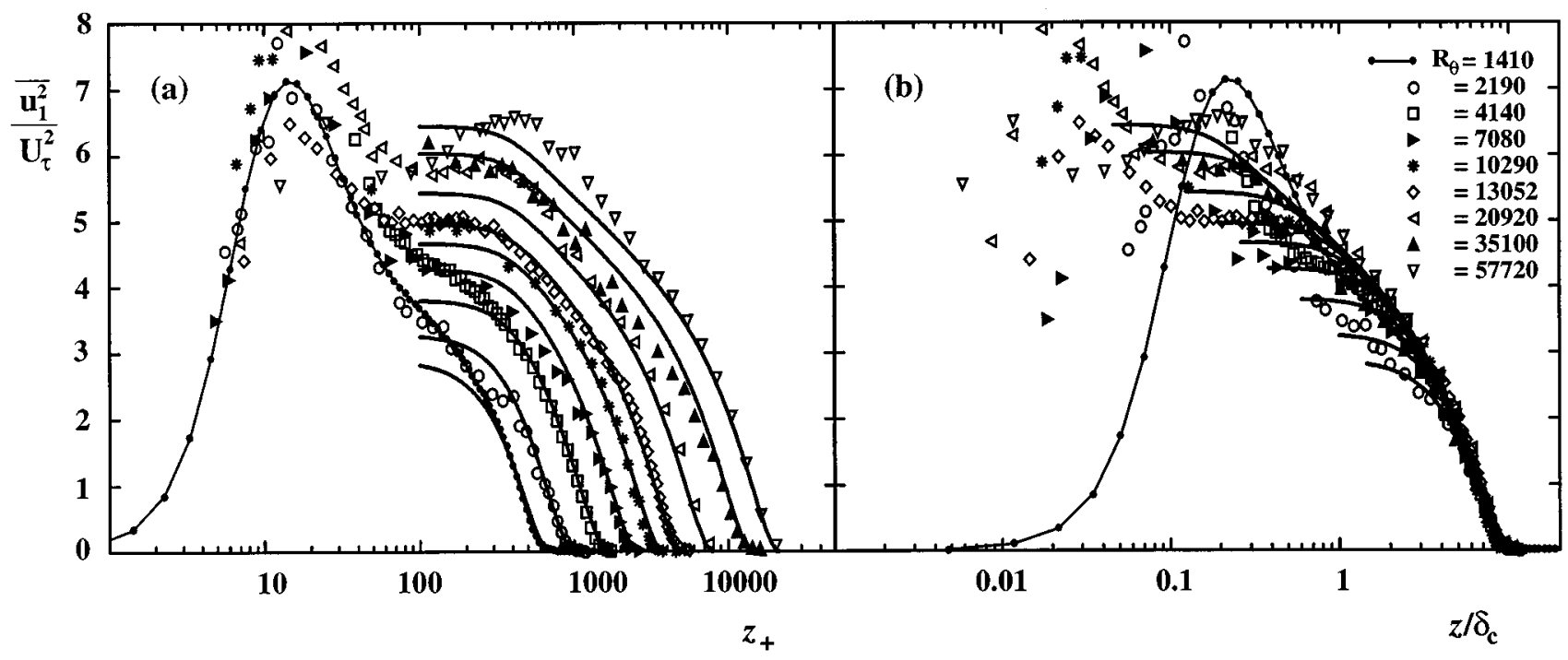

FIG. 5. A collection of experimental streamwise broadband turbulence intensity profiles together with similarity predictions given by equation (8) (solid lines). (a) Inner-flow scaling. (b) Outer-flow scaling. Corresponding $K_{\tau}$ values are given in the Appendix. Sources of the data are as follows: $R_{\theta}=1410-$ Spalart; 2190, 7080, 10290-Mclean; 4140—Uddin; 13052—Smith; 20920, 57720—DNW; 35100-Petrie et al.

However, all of this was done at low Reynolds numbers and what is also needed are accurate measurements at moderate to high Reynolds numbers. Unfortunately, the size of the viscous zone becomes increasingly small if the Reynolds number variation is carried out by increasing $U_{\infty}$ or by decreasing $\nu$ and this will introduce spatial and temporal resolution problems. Measurements in thick boundary layers (i.e., an increase in length scale) achieved through long development lengths may be a way to tackle these problems.

Apart from being more applicable in practice, studying high Reynolds number flows is important in order to adequately test theories such as the attached eddy hypothesis. This extends to discussions of what Bradshaw ${ }^{32}$ and Townsend ${ }^{11}$ refer to as "inactive motions." These are largescale motions which contribute to the streamwise and spanwise broadband-turbulence intensities near the wall but not the wall-normal turbulence intensity and Reynolds shear

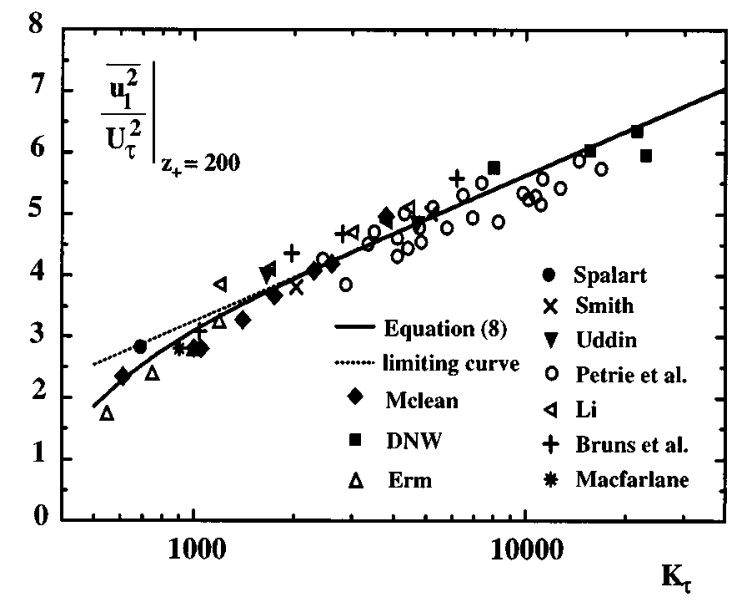

FIG. 6. The streamwise broadband turbulence intensity at $z_{+}=200$ for a range of different $K_{\tau}$ values (see the Appendix). Solid line is obtained from the similarity formulation (8). stress. Townsend ${ }^{11}$ points out that these "motions" can be fully described by the presence of attached eddies. The attached eddy model ${ }^{17,13}$ describes the boundary layer by superimposing the contributions from a range a scales of geometrically similar representative eddies, and the contribution of one scale of eddies of length scale $\delta$ to $\overline{u_{1}^{2}} / U_{\tau}^{2}$ is proportional to $I_{11}[z / \delta]$, the streamwise Townsend eddy intensity function. By Taylor series expanding the velocity field for inviscid boundary conditions, Townsend ${ }^{12}$ deduced that $I_{11}$ must approach a constant as $z / \delta \rightarrow 0$, whereas it rapidly decays to zero for increasing $z / \delta$ for $z / \delta>1$. This directly implies that all eddies with length scale $\delta>O(z)$ will have contributions to $\overline{u_{1}^{2}} / U_{\tau}^{2}$ at this $z$ position and this results in the "inactive motions." This also applies to the intensity function $I_{22}[z / \delta]$, and hence $\overline{u_{2}^{2}} / U_{\tau}^{2}$, the spanwise intensity. This is not true for $I_{33}$ and $I_{13}$ which fall to zero as $z / \delta \rightarrow 0$. As the Reynolds number increases, the range of eddy length scales $\delta$ increases and so $\overline{u_{1}^{2}} / U_{\tau}^{2}$ and $\overline{u_{2}^{2}} / U_{\tau}^{2}$ keep increasing but $\overline{u_{3}^{2}} / U_{\tau}^{2}$ and $-\overline{u_{1} u_{3}} / U_{\tau}^{2}$ asymptote to a finite universal limit above the buffer zone. The detailed mathematics behind this has been given in many places, e.g., Perry and Marusic, ${ }^{13}$ Perry et al., ${ }^{17}$ Perry and Chong, ${ }^{16}$ Townsend $^{12}$ and Smits and Dussauge ${ }^{5}$ to mention a few and therefore will not be reproduced here. It is important to restate that this only applies for the region above the viscous buffer zone where inviscid boundary conditions apply and the motions can be thought of as a "meandering and swirling" of the fluid in the streamwise-spanwise plane.

Within the viscous sublayer and buffer zone, where the eddy formation processes are taking place, it is not possible to draw firm conclusions about the influence of these "inactive motions," but it would seem reasonable to speculate the following. Since the intensities $\overline{u_{1}^{2}} / U_{\tau}^{2}$ and $\overline{u_{2}^{2}} / U_{\tau}^{2}$ increase with increasing Reynolds number above the buffer zone it is likely that these components will not show inner scaling in the viscous sublayer and buffer zone. Conversely, since 
$\overline{u_{3}^{2}} / U_{\tau}^{2}$ and $-\overline{u_{1} u_{3}} / U_{\tau}^{2}$ retain universal values invariant with $z_{+}$for $z / \delta_{c} \rightarrow 0$ above the buffer zone for increasing Reynolds numbers, it is likely that these quantities will show inner scaling in the viscous sublayer and buffer zone. None of this is likely to be valid if the Reynolds number is not sufficiently large, since the ratio of the largest to smallest attached eddies is proportional to $K_{\tau}=\delta_{c} U_{\tau} / \nu$, and restricting measurements to low-Reynolds-number flows may mean This is probably the case for the low-Reynolds-number data reviewed by Bradshaw and Huang ${ }^{7}$ where they concluded that none of the Reynolds stresses show inner-scaling. Further experiments in moderate to high Reynolds numbers are needed to resolve the issue.

\section{B. Outer region}

In the region of the flow beyond the buffer zone, the results in Fig. 5(a) show clear Reynolds number effects for data plotted against $z_{+}$. Coles ${ }^{4}$ also concluded that beyond the buffer zone $\overline{u_{1}^{2}} / U_{\tau}^{2}$ should increase with increasing Reynolds number at a fixed $z_{+}$. To investigate this trend further, Fig. 6 shows how the streamwise broadband-turbulence intensity varies with $\delta_{c} U_{\tau} / \nu$ at $z_{+}=200$ for the similarity formulation and for various experimental studies. Reasonably good agreement is observed. The similarity formulation is seen to approach a $\log$-linear relationship (i.e., negligible $V\left[z_{+}\right]$effects) beyond $K_{\tau} \approx 2500$.

The results in Fig. 6 indicate that the similarity proposed will result in the plateau, observed in the $\overline{u_{1}^{2}} / U_{\tau}^{2}$ versus $\log \left[z_{+}\right]$at $z_{+}=O(100)$, to rise with Reynolds number without limit. How far this formulation can be pushed is not known. It would be interesting to see whether this level ultimately exceeds the peak turbulence intensity at $z_{+} \approx 15$. Also if the trend shown in Fig. 6 were to continue with increasing $K_{\tau}$ then $\left(\overline{u_{1}^{2}} / U^{2}\right)_{z_{+}}=200$ (where $U$ is the local mean velocity) will increase without limit. This ultimately must lead to momentary flow reversals occurring. At a first glance, it would appear that the formulation might "self dethis. Nevertheless, from work by Perry and Chong ${ }^{33}$ which considers the flow topology of turbulent boundary layers near the wall, momentary flow reversals were shown to occur even in the Spalart ${ }^{21}$ zero-pressure-gradient flow case, at least at $z_{+} \rightarrow 0$. These observations come from studying streamline and vortex line patterns. The same work also shows that in adverse-pressure-gradients layers such momentary flow reversals in localized regions are very common as seen in the data of Spalart and Watmuff ${ }^{34}$ at least at $z_{+} \rightarrow 0$ even though the flow in the mean is attached. It could well be that the similarity law is still valid even with such flow reversals.

Another interesting feature in Fig. 5 is the high Reynolds number data of Fernholz et al. ${ }^{35}$ (labelled DNW). Here an additional peak appears to be present in the outer part of the flow. The data of Petrie et al. ${ }^{36}$ at a similar Reynolds number do not show this trend. Unfortunately there is very little experimental data at these high Reynolds numbers with which to investigate this behavior further. For this issue and many that an inadequate range of scales of attached eddies exists. struct" at sufficiently high Reynolds numbers because of

others it is certainly desirable to have systematic studies in very thick high Reynolds number boundary layers. The authors are presently engaged in constructing a facility for producing such layers.

\section{SUMMARY AND CONCLUSIONS}

A similarity formulation has been proposed providing a framework with which to describe Reynolds number effects on $\overline{u_{1}^{2}} / U_{\tau}^{2}$ for the entire region above the viscous buffer zone (i.e., $z_{+} \geqslant 100$ and $z / \delta_{c} \leqslant 1$ ) for flows with zero pressure gradient, provided $R_{\theta}>6000$ approximately. For lower Reynolds numbers, the lower limit of $z_{+}$increases as $R_{\theta}$ decreases. The formulation, which is completely consistent with attached eddy model given by Perry and Marusic ${ }^{13}$ and Marusic and Perry, ${ }^{14}$ bears an analogy with the mean-flow velocity profile. It includes a logarithmic relationship in the turbulent wall region with deviations at the two ends: one near the buffer zone and the other in the wake region. These deviations are referred to as the viscous deviation and the wake deviation. The wake deviation is based on Townsend's Reynolds-number-similarity hypothesis and is thus a universal function of $z / \delta_{c}$ for a fixed Coles wake factor $\Pi$. The viscous deviation is proposed to be made up of an isotropic Kolmogorov cut-off component and anisotropic components which could include a cut-off of anisotropic dissipating motions and an attached eddy cut-off which is consistent with the attached eddy model. ${ }^{17,13}$

The formulation is compared to experimental data covering a large range of Reynolds number including new data presented here and other data not reviewed previously. Encouraging agreement is observed for flows with moderate to high Reynolds numbers thus supporting the attached eddy hypothesis and outer-flow Reynolds number similarity.

The formulation does not extend into the mean-flow viscous buffer zone and sublayer but discussion is given about inner-scaling and the need for further experiments in the near-wall region of moderate and high Reynolds number flows.

\section{ACKNOWLEDGMENT}

The authors wish to gratefully acknowledge the financial assistance of the Australian Research Council.

\section{APPENDIX: $\boldsymbol{R}_{\theta}$ AND $K_{\tau}$ VALUES FOR DIFFERENT FLOW CASES}

\begin{tabular}{lcc}
\multicolumn{1}{c}{ Data source } & $R_{\theta}$ & $K_{\tau}$ \\
\hline $\begin{array}{l}\text { Authors' data }\left(\mathrm{Uddin}^{26}\right) \\
\text { (HWA) }\end{array}$ & 4140 & 1655 \\
& 11928 & 4704 \\
Bruns et al. & 37 & \\
(HWA) & 2573 & 1035 \\
& 5023 & 1970 \\
& 7140 & 2805 \\
& 16080 & 6200 \\
Erm and Joubert & & \\
(HWA) & 1003 & 547 \\
& 1568 & 749
\end{tabular}


Fernholz et al. ${ }^{35}$ (DNW)

(HWA)

20920

41260

57720

60810

Mclean $^{38}$

(HWA)

1500

2190

2670

3860

4950

4980

7080

10290

Perry and $\mathrm{Li}^{10}$

(HWA)

2828

4066

7357

9371

11097

$R_{\theta}$

Data source

Macfarlane ${ }^{39}$

(HWA)

Petrie et $a l .{ }^{36}$

(LDA)

2027

6570

7990

9130

9280

10000

10500

10740

10960

11200

13100

13540

14000

15400

16700

18300

21700

23400

24300

24301

26000

26200

28600

35100

39000

Smith $^{40}$

(HWA)

5021

13052

Spalart $^{21}$

(DNS)

1410
8008

15643

21603

23013

610

1000

1050

1400

1750

2300

2600

3800

1193

1681

3000

3780

4433

$K_{\tau}$

903

2439

2864

3349

3497

4411

4096

4088

4301

4846

5244

4775

5771

6467

6915

8293

7346

9862

10148

11154

11235

10749

12732

14455

16850

2030

5180

689

${ }^{1}$ M. Gad el Hak and P. R. Bandyopadhyay, "Reynolds number effects in wall-bounded turbulent flows," Appl. Mech. Rev. 47 (8), 307-365 (1994).

${ }^{2}$ H. H. Fernholz and P. J. Finley, "The incompressible zero-pressuregradient turbulent boundary layer: An assessment of the data," Prog. Aerospace Sci. 32, 245-311 (1996).

${ }^{3}$ S. Mochizuki and F. T. M. Nieuwstadt, "Reynolds-number-dependence of the maximum in the streamwise velocity fluctuations in wall turbulence," Exp. Fluids 21, 218-226 (1996).

${ }^{4}$ D. Coles, "A model for flow in the viscous sublayer," in Proc. Workshop on Coherent Structure of Turbulent Boundary Layers, edited by C. R Smith and D. E. Abbott (Lehigh University, 1978).

${ }^{5}$ A. J. Smits and J. P. Dussauge, Turbulent Shear Layers in Supersonic Flow (AIP, New York, 1996).

${ }^{6}$ J. P. Dussauge, H. Fernholz, P. J. Finley, R. W. Smith, A. J. Smits, and E. F. Spina, "Turbulent boundary layers in subsonic and supersonic flow," AGARDograph, 335 (1996).

${ }^{7}$ P. Bradshaw and G. P. Huang, "The law of the wall in turbulent flow," Proc. R. Soc. London, Ser. A 451, 165-188 (1995).

${ }^{8}$ F. Durst, J. Jovanovic, and J. Sender, "LDA measurements in the nearwall region of a turbulent pipe flow," J. Fluid Mech. 295, 305-335 (1995).

${ }^{9}$ A. E. Perry, K. L. Lim, and S. M. Henbest, “An experimental study of the turbulence structure in smooth- and rough-wall boundary layers,' J. Fluid Mech. 177, 437-466 (1987).

${ }^{10}$ A. E. Perry and J. D. Li, "Experimental support for the attached eddy hypothesis in zero-pressure-gradient turbulent boundary layers," J. Fluid Mech. 218, 405-438 (1990).

${ }^{11}$ A. A. Townsend, "Equilibrium layers and wall turbulence," J. Fluid Mech. 11, 97-120 (1961).

${ }^{12}$ A. A. Townsend, The Structure of Turbulent Shear Flow, Vol. 2 (Cambridge University Press, Cambridge, 1976).

${ }^{13}$ A. E. Perry and I. Marusic, "A wall-wake model for the turbulence structure of boundary layers. Part 1. Extension of the attached eddy hypothesis," J. Fluid Mech. 298, 361-388 (1995).

${ }^{14}$ I. Marusic and A. E. Perry, "A wall-wake model for the turbulence structure of boundary layers. Part 2. Further experimental support," J. Fluid Mech. 298, 389-407 (1995).

${ }^{15}$ S. J. Kline, W. C. Reynolds, F. A. Schaub, and P. W. Rundstadler, “The structure of turbulent boundary layers,' J. Fluid Mech. 30, 741-773 (1967).

${ }^{16}$ A. E. Perry and M. S. Chong, "On the mechanism of wall turbulence," J. Fluid Mech. 119, 173-217 (1982).

${ }^{17}$ A. E. Perry, S. M. Henbest, and M. S. Chong, "A theoretical and experimental study of wall turbulence,", J. Fluid Mech. 165, 163-199 (1986).

${ }^{18}$ A. E. Perry and C. J. Abell, "Asymptotic similarity of turbulence structures in smooth- and rough-walled pipes," J. Fluid Mech. 79, 785-799 (1977).

${ }^{19}$ L. S. G. Kovasznay, “Spectrum of locally isotropic turbulence,' J. Aerosol Sci. 15, 745-753 (1948).

${ }^{20}$ A. E. Perry, J. D. Li, S. M. Henbest, and I. Marusic, "The attached eddy hypothesis in wall turbulence," in Near-Wall Turbulence (1988 Zoran Zaric Memorial Conference), Dubrovnik, Croatia (Hemisphere, New York, 1988).

${ }^{21} \mathrm{P}$. R. Spalart, "Direct simulation of turbulent boundary layer up to $R_{\theta}=1410, "$ J. Fluid Mech. 187, 61-98 (1988).

${ }^{22}$ S. H. M. Hafez, "The structure of accelerated turbulent boundary layers," Ph.D. thesis, University of Melbourne, Australia, 1991.

${ }^{23}$ A. E. Perry, I. Marusic, and J. D. Li, "Wall turbulence closure based on classical similarity laws and the attached eddy hypothesis,' Phys. Fluids 2(6), 1024-1035 (1994).

${ }^{24}$ D. E. Coles, "The law of the wake in the turbulent boundary layer," $\mathrm{J}$. Fluid Mech. 1, 191-226 (1956).

${ }^{25}$ A. E. Perry, Hot-wire Anemometry (Clarendon Press, Oxford, 1982).

${ }^{26}$ A. K. M. Uddin, "The structure of a turbulent boundary layer," Ph.D. thesis, University of Melbourne, Australia, 1994.

${ }^{27}$ A. A. Townsend, The Structure of Turbulent Shear Flow (Cambridge University Press, Cambridge, 1956).

${ }^{28}$ R. A. Antonia, S. Rajagopalan, C. S. Subramanian, and A. J. Chambers, "Reynolds-number dependence of the structure of a turbulent boundary layer,' J. Fluid Mech. 121, 123-140 (1982).

${ }^{29}$ L. P. Erm and P. N. Joubert, "Low Reynolds number turbulent boundary layers," J. Fluid Mech. 230, 1-44 (1991).

${ }^{30}$ A. K. Lewkowicz, "An improved universal wake function for turbulent boundary layers and some of its consequences,' Z. Flugwiss. Weltraumforsch 6, 261-266 (1982).

${ }^{31}$ W. R. C. Phillips, "The wall region of the turbulent boundary layer," Phys. Fluids 30 (8), 2354-2361 (1987).

${ }^{32} \mathrm{P}$. Bradshaw, "The turbulence structure of equilibrium boundary layers," J. Fluid Mech. 29, 625-645 (1967).

${ }^{33}$ A. E. Perry and M. S. Chong, "Topology of flow patterns in vortex mo- 
tions and turbulence,' Appl. Sci. Res. 53, 357-374 (1994).

${ }^{34}$ P. R. Spalart and J. H. Watmuff, "Experimental and numerical study of turbulent boundary layers with pressure gradients,', J. Fluid Mech. 249, 271-298 (1992).

${ }^{35}$ H. H. Fernholz, E. Krause, M. Nockemann, and M. Schober, 'Comparative measurements in the canonical boundary layer at $R e_{\theta} \leqslant 6 \times 10^{4}$ on the wall of the DNW,', Phys. Fluids 7, 1275-1281 (1995).

${ }^{36}$ H. L. Petrie, A. A. Fontaine, S. T. Sommer, and T. A. Brungart, "Large flat plate turbulent boundary layer evaluation," Tech. Rep. TM89-207, Appl. Res. Lab., Penn State University, 1990.

${ }^{37}$ J. Bruns, P. Dengel, and H. H. Fernholz, "Mean flow and turbulence measurements in a an incompressible two-dimensional turbulent boundary layer. Part I: Data,' Technical Report Institutsbericht Nr. 02/92, HermanFöttinger-Institut für Thermound Fluiddynamik, Technische Universität Berlin, 1992.

${ }^{38}$ I. R. McLean, "The near wall eddy structure in an equilibrium turbulent boundary layer,' 'Ph.D. thesis, University of Southern California, USA, 1990.

${ }^{39}$ I. Macfarlane, "Turbulent boundary layers with system rotation," Ph.D. thesis, University of Melbourne, Australia, 1996.

${ }^{40}$ R. W. Smith, "The effect of Reynolds number on the structure of turbulent boundary layers,', Ph.D. thesis, Princeton University, 1994. 


\section{University Library}

\section{- M M I N E R VA A gateway to Melbourne's research publications}

Minerva Access is the Institutional Repository of The University of Melbourne

Author/s:

Marusic, I.;Uddin, A. K. M.;Perry, A. E.

Title:

Similarity law for the streamwise turbulence intensity in zero-pressure-gradient turbulent boundary layers

Date:

1997

Citation:

Marusic, I., Uddin, A. K. M. \& Perry, A. E. (1997). Similarity law for the streamwise turbulence intensity in zero-pressure-gradient turbulent boundary layers. Physics of Fluids, 9(12), 3718-3726.

Publication Status:

Published

Persistent Link:

http://hdl.handle.net/11343/34793 